\title{
IMPACTO DE TEMPESTADE GEOMAGNÉTICA NA \\ IONOSFERA E NO POSICIONAMENTO COM GNSS: \\ ESTUDO DE CASO PARA 20 DE NOVEMBRO DE 2003 NA \\ REGIÃO BRASILEIRA
}

Impact of Geomagnetic Storm in the Ionosphere and GNSS Positioning: Study of case for November 20, 2003 in Brazilian region.

\author{
MARCELO TOMIO MATSUOKA ${ }^{1}$ \\ CAROLINA COLLISCHONN ${ }^{1}$ \\ IVANDRO KLEIN $^{1}$ \\ PAULO DE OLIVEIRA CAMARGO ${ }^{2}$ \\ VINICIUS AMADEU STUANI PEREIRA ${ }^{2}$
}

${ }^{1}$ Laboratório de Pesquisas em Geodésia (LAGEO)/Departamento de

Geodésia/Instituto de Geociências - UFRGS

${ }^{2}$ Departamento de Cartografia/Faculdade de Ciências e Tecnologia/Universidade

Estadual Paulista - FCT/Unesp

carol.collischonn@gmail.com; tomiomatsuoka@gmail.com;

ivandroklein@gmail.com; paulo@fct.unesp.br; vi_stuani@hotmail.com

\begin{abstract}
RESUMO
O objetivo deste artigo é analisar a influência de intensas tempestades geomagnéticas na ionosfera e no posicionamento por GNSS (GPS). Neste sentido, foram analisados os efeitos da intensa tempestade geomagnética de 20 de novembro de 2003 utilizando dados GPS de algumas estações da RBMC (Rede Brasileira de Monitoramento Contínuo), situadas em diferentes locais na região brasileira, e mapas globais da ionosfera. Analisando os resultados se observa um aumento na densidade de elétrons livres da ionosfera nas regiões mais próximas do equador geomagnético no período da tarde no dia da tempestade. No período após o pôr do Sol do dia da tempestade, verifica-se um aumento na densidade de elétrons livres e de irregularidades ionosféricas em regiões mais afastadas do equador geomagnético, em comparação com dias geomagneticamente calmos. Quando analisado o posicionamento por ponto, se observa maiores discrepâncias em planimetria e altimetria no posicionamento para os mesmos períodos em que ocorreram mudanças
\end{abstract}


na ionosfera, principalmente para as estações GPS mais afastadas do equador geomagnético no período após o pôr do Sol.

Palavras-chave: GNSS; GPS; Ionosfera; Tempestade Geomagnética; TEC.

\begin{abstract}
The goal of this paper is to analyze the influence of the intense geomagnetic storms in the ionosphere and GNSS (GPS) positioning. It was analyzed the effects of intense geomagnetic storm of November 20th 2003 using GPS data from RBMC (Brazilian Network for Continuous Monitoring) located in different sites in the Brazilian region and ionosphere global maps. While analyzing the results, it can be observed an increase in the electron density of the ionosphere in the regions near to the geomagnetic equator in the afternoon on the day of the storm. In the period after the sunset of the storm day, there is an increase in the density of free electrons and ionospheric irregularities in regions furthest from the geomagnetic equator, when compared to geomagnetically quiet days. When the positioning point is analyzed, it is observed high discrepancies values in planimetry and altimetry at the same position for periods of changes that occurred in the ionosphere, especially for the GPS stations located furthest from the geomagnetic equator in the period after the sunset Sun.
\end{abstract}

Keywords: GNSS; GPS; Ionosphere; Geomagnetic Storm; TEC.

\title{
1. INTRODUÇÃO
}

Entre as diferentes fontes de erros que afetam o posicionamento com receptores GNSS (Global Navigation Satellite Systems) de uma frequência uma das principais é a ionosfera. A ionosfera é a camada da atmosfera terrestre compreendida entre $50 \mathrm{~km}$ e $1000 \mathrm{~km}$ de altitude, aproximadamente, onde existe a presença de elétrons livres em quantidade suficiente para afetar a propagação de ondas de rádio (DAVIES, 1990). O principal processo de formação de elétrons livres na ionosfera é a fotoionização, que consiste na absorção de radiação solar, predominantemente na faixa do extremo ultravioleta e raios $\mathrm{X}$, por elementos atmosféricos neutros (KIRCHHOFF, 1991). Dessa forma, o comportamento da ionosfera é fortemente influenciado pela atividade solar.

$\mathrm{O}$ erro associado à ionosfera nas observáveis GNSS é proporcional ao conteúdo total de elétrons livres (TEC - Total Electron Content) presente na trajetória do sinal entre o satélite e a antena receptora. O TEC, e consequentemente o erro devido à ionosfera, varia no tempo e no espaço principalmente com relação à variação da radiação solar e do campo magnético da Terra. O Brasil é uma das regiões do globo terrestre que possuem os maiores valores e variações do TEC (KOMJATHY et al., 2003). Estudos pioneiros que mostraram que as medidas GPS (Global Positioning System) na região brasileira são afetadas por diferentes condições ionosféricas, podem ser verificados em Wanninger et al. (1991) e Campos et al. (1993). 
Além das variações regulares, o TEC pode sofrer abruptas modificações em seu comportamento devido à ocorrência de eventos solares, tais como, intensas explosões solares (solar flares). Durante explosões solares, um primeiro fenômeno que ocorre é o aumento da radiação solar eletromagnética na faixa do comprimento de onda dos raios X. Se a explosão solar for geoefetiva, na direção da Terra, pode provocar uma série de fenômenos na ionosfera que genericamente são chamados de Distúrbios Ionosféricos Súbitos (DIS) (DAVIES, 1990).

Entre os DIS que podem ocorrer, está o rápido aumento da densidade de elétrons na ionosfera, ou seja, do TEC. Exemplos de estudos deste tipo de fenômeno e seus efeitos no posicionamento por GNSS podem ser verificados, por exemplo, em Afraimovich et al. (2001), Liu et al. (2006), Matsuoka et al. (2006).

Outro fenômeno que pode acontecer quando ocorrem explosões solares é a ejeção de massa coronal (CME - Coronal Mass Ejection) (McNAMARA, 1991). Esse evento pode ocasionar perturbações no campo geomagnético e, quando muito intensa, ocasiona tempestades geomagnéticas que implicam em tempestades ionosféricas. Tempestades geomagnéticas/ionosféricas afetam o comportamento do TEC e, consequentemente, o posicionamento com GNSS. Efeitos de tempestades geomagnéticas na ionosfera e/ou no posicionamento com GNSS na região brasileira, podem ser verificados, por exemplo, em Becker-Guedes et al. (2007), Matsuoka et al. (2008), Salomoni et al. (2011).

Nesse artigo são apresentados e discutidos os resultados obtidos de experimentos realizados com o objetivo de verificar a influência da intensa tempestade geomagnética de 20 de novembro de 2003 no posicionamento por ponto GPS (Global Positioning System) na região brasileira, utilizando dados de estações da RBMC (Rede Brasileira de Monitoramento Continuo) do IBGE (Instituto Brasileiro de Geografia e Estatística), e a relação com a atividade solar e ionosférica.

Vale salientar que atualmente está se iniciando o período de maior ocorrência de intensos fenômenos solares do ciclo solar 24 e, portanto, estudos anteriores dos efeitos de tempestades geomagnéticas intensas do ciclo anterior auxiliam no entendimento do que se pode esperar para as futuras tempestades.

\section{VARIAÇÕES DO TEC}

As variações temporais regulares do TEC compreendem as variações diurnas, sazonais e ciclos de longos períodos. A variação diurna ocorre principalmente devido à radiação solar. Ao longo do dia, a densidade de elétrons depende da hora local, sendo que seu valor máximo ocorre entre as 12 e 16 horas local (WEBSTER, 1993). Na região equatorial de baixas latitudes um segundo pico ocorre nas horas que precedem a meia noite, principalmente, em períodos próximos aos equinócios e verão e em períodos de alta atividade solar.

As estações do ano também influenciam na variação da densidade de elétrons, devido à mudança do ângulo zenital do Sol e da intensidade do fluxo de ionização, caracterizando as variações sazonais. Nos equinócios, os efeitos da ionosfera são 
maiores, enquanto que, nos solstícios, os efeitos são menores. Isto pode ser verificado, por exemplo, em experimentos realizados por Matsuoka e Camargo (2004).

As variações de ciclos de longos períodos, cada ciclo com duração de aproximadamente 11 anos, são associadas às ocorrências de manchas solares, e o aumento de ionização, ou seja, o aumento do TEC é proporcional ao número de manchas. O ciclo solar atual é o 24 que se iniciou em meados de 2008.

A localização geográfica também influencia na variação da densidade de elétrons na ionosfera, pois a estrutura global da ionosfera não é homogênea. Ela muda com a latitude, devido à variação do ângulo zenital do Sol, que influencia, diretamente, no nível de radiação que altera, por sua vez, a densidade de elétrons na ionosfera. As regiões equatoriais são caracterizadas por um alto nível de densidade de elétrons, bem como, apresentam uma alta variação espacial. Já as regiões de latitudes médias são consideradas relativamente livres das anomalias ionosféricas, apresentando um comportamento mais regular e previsível. A ionosfera sobre os pólos sul e norte, alternativamente denominadas de ionosfera polar ou de altas latitudes, é extremamente instável (McNAMARA, 1991).

Mais detalhes sobre as variações regulares do TEC podem ser obtidos, por exemplo, em McNamara (1991), Davies (1990), Camargo (1999) e Matsuoka (2007).

\subsection{Anomalia Equatorial de Ionização}

Antes de descrever a Anomalia de Ionização Equatorial (AIE) é necessário entender o fenômeno que a origina. A interação entre os campos elétrico e magnético da Terra com a grande quantidade de íons positivos e elétrons livres na região equatorial, criados pelo processo de fotoionização no lado diurno, gera uma movimentação do plasma ao longo das linhas de força horizontais do campo geomagnético para longe do equador.

Os campos elétricos do dínamo atmosférico que são gerados na camada $\mathrm{E}$ são transmitidos ao longo das linhas de campo geomagnético para a camada $\mathrm{F}$, devido à alta condutividade paralela. Durante o dia o campo elétrico (E) é direcionado para leste. Na camada $\mathrm{F}$ equatorial, um campo elétrico para leste, na presença do campo magnético (B) que é dirigido para norte causa uma deriva eletromagnética para cima, dada por $\mathrm{ExB} / \mathrm{B}^{2}$. Assim o plasma é lançado até elevadas altitudes na região equatorial, e após isso ele desce ao longo das linhas de campo geomagnético para o norte e para o sul devido à ação da gravidade e do gradiente de pressão. Este movimento de elevação do plasma na região equatorial e posterior descida ao longo das linhas de campo geomagnético até baixas latitudes é conhecido como Efeito Fonte (APPLETON, 1946).

Como consequência da subida e posterior descida do plasma se formam duas cristas, onde esses picos de densidade de elétrons nas regiões subtropicais ao norte e ao sul do equador geomagnético encontram-se, aproximadamente, em $15^{\circ}$ de latitude geomagnética. Em contrapartida, no equador geomagnético a densidade de 
elétrons fica menor. Essa distribuição latitudinal da densidade de elétrons livres é denominada de Anomalia Equatorial de Ionização, doravante no texto apenas será chamada de anomalia equatorial. Devido à relação com o campo geomagnético, períodos de tempestades geomagnéticas podem alterar o comportamento da anomalia equatorial.

\subsection{Irregularidades Ionosféricas}

As irregularidades ionosféricas são perturbações na densidade do plasma ionosférico e se originam por processos de instabilidade do plasma que ocorrem na ionosfera equatorial. Essa instabilidade dá origem às irregularidades na camada $\mathrm{F}$ ionosférica com amplo espectro de escalas de tamanho, desde alguns centímetros até centenas de quilômetros (RODRIGUES, 2003).

Irregularidades na ionosfera podem produzir variações de curtos períodos nos sinais trans-ionosféricos, provocadas por rápidas flutuações na fase e amplitude, causando um enfraquecimento e variações da fase no sinal recebido pelos receptores GNSS, podendo provocar, em alguns casos, perdas do sinal (WEBSTER, 1993). Essas rápidas flutuações em fase e amplitude do sinal são chamadas de cintilações.

Vários estudos sobre irregularidades ionosféricas na região brasileira já foram realizados. Por exemplo, em um estudo realizado por Skone et al. (2001) utilizando dados GPS, observou-se que os efeitos de irregularidades ionosféricas nos sinais GPS são mais presentes após o pôr do Sol nos meses de setembro a março e em períodos de alta atividade solar. Além disso, verificou-se também que a cintilação ionosférica tem relação com a anomalia equatorial, sendo observada maior intensidade nos locais mais próximos das cristas da anomalia equatorial (nos picos de densidade de elétrons da anomalia equatorial).

Com valores de TEC obtidos com dados GNSS de receptores de dupla frequência pode-se estudar as irregularidades ionosféricas. Isto porque a taxa de variação do TEC é um indicador da ocorrência de irregularidades na ionosfera. Diversos índices de irregularidades ionosféricas foram desenvolvidos a partir de valores de variação do TEC obtidos de dados GNSS dos quais podem ser verificados alguns, por exemplo, em Wanninger (1993), Pi et al. (1997) e Mendillo et al. (2000).

Tempestades geomagnéticas podem provocar mudanças no comportamento das irregularidades ionosféricas numa determinada região, podendo aumentar ou até mesmo suprimir a ocorrência de irregularidades.

\section{TEMPESTADES GEOMAGNÉTICAS}

O campo magnético da Terra exerce grande influência na variação da densidade de elétrons na ionosfera. $\mathrm{O}$ campo geomagnético controla o movimento das partículas ionizadas e, portanto, qualquer perturbação no campo geomagnético resultará em modificações nas condições de transporte do meio ionizado.

Além da radiação eletromagnética, o Sol também libera um fluxo de partículas carregadas chamado de vento solar (KIRCHHOFF, 1991). O vento solar ocorre 
continuamente, confinando e distorcendo o campo magnético da Terra em uma cavidade com uma cauda longa que se estende por centenas de raios terrestres na direção anti-solar (FEDRIZZI, 2003), fazendo com que o campo geomagnético se torne diferente do conhecido modelo teórico de Dipolo. Essa cavidade denomina-se magnetosfera.

Durante eventos solares intensificados, que por sua vez intensificam o vento solar, a velocidade do mesmo pode passar de 300-400 para $1000 \mathrm{Km} / \mathrm{s}$ ou até mais. Dependo da intensidade da perturbação, o limite da magnetopausa (linha de contorno da magnetosfera) é comprimido, podendo passar de 10 a 6 raios terrestres (FEDRIZZI, 2003). Em outras palavras, eventos solares são responsáveis pela alteração do campo geomagnético, que por sua vez contribuem para a ocorrência das tempestades geomagnéticas. Estas por sua vez, implicam numa tempestade ionosférica, que é uma resposta à tempestade geomagnética.

Os eventos solares que causam tempestades geomagnéticas são: explosões solares (solar flares), corrente de vento solar de alta velocidade (HSSWS - High Speed Solar Wind Stream) provenientes de buracos na coroa solar (coronal holes) e desaparecimento súbito de filamentos (SDF's - Sudden Disappearing Filaments) (McNAMARA, 1991). De acordo com Gonzáles et al. (1999), as explosões solares e os SDF's são fenômenos de períodos de alta atividade solar, e os buracos na coroa estão presentes durante períodos de baixa e média atividade.

As explosões solares ocorrem em regiões do Sol denominadas ativas onde se encontram manchas solares (McNAMARA, 1991). A duração de uma explosão solar é de cerca de 3 minutos até várias horas, sendo o tempo médio de aproximadamente 30 minutos (DAVIES, 1990). Durante explosões solares um dos fenômenos que ocorre é o aumento da radiação solar eletromagnética na faixa do comprimento de onda dos raios X (McNAMARA, 1991), provocando um aumento muito rápido na ionização (pelo processo de fotoionização) no lado iluminado da Terra. Esse aumento de ionização dá origem a uma série de fenômenos, denominados genericamente de "Distúrbios Ionosféricos Súbitos" (DIS). Segundo Sandford (1991), durante eventos de DIS podem ocorrer rápidas variações na amplitude e fase dos sinais GNSS, podendo até ocorrer perdas de sinal.

Durante explosões solares muito intensas, outro fenômeno que pode ocorrer é a ejeção de uma corrente (fluxo) de prótons que pode alcançar a Terra no caso de ser ejetado na direção dela (McNAMARA, 1991). Quando os prótons chegam a Terra eles encontram o campo magnético terrestre. Sendo uma partícula carregada, um próton não pode cruzar as linhas de força do campo geomagnético, devendo girar ao redor delas. As linhas de força do campo geomagnético são horizontais próximas ao equador e verticais próximas aos pólos. Isto implica que prótons na direção do equador não podem penetrar diretamente na ionosfera, e dessa forma, a ionosfera equatorial é livre dos efeitos dos prótons provenientes de explosões solares. Já no caso das regiões polares, por terem linhas de força do campo geomagnético verticais, os prótons girando ao redor dessas linhas, penetram diretamente na ionosfera polar (McNAMARA, 1991). 
Se a explosão solar for muito intensa, tem-se o terceiro fenômeno: a ejeção de uma "nuvem" de plasma, conhecido também como ejeção de massa coronal (CME Coronal Mass Ejection) (DAVIES, 1990; McNAMARA, 1991). Ejeção de massa coronal é a denominação dada a grandes quantidades de plasma que são subitamente expelidas a partir do Sol. Os parâmetros do vento solar são drasticamente intensificados durante esse fenômeno, podendo causar uma tempestade geomagnética/ionosférica (McNAMARA, 1991).

Nem todas as CME's ocorrem na direção da Terra, sendo que as que ocorrem são chamadas de CME's Halo (HATHAWAY, 2007). As tempestades geomagnéticas mais intensas são frequentemente relacionadas à ocorrência de uma CME.

\section{1 Índices Geomagnéticos}

Índices Geomagnéticos constituem uma série de dados que visam descrever em escala planetária a atividade geomagnética ou alguns de seus componentes (ISGI, 2011). Dentre os índices mais usados para determinar a intensidade e a duração das tempestades geomagnéticas está o índice Kp (Planetarische Kennziffer), do qual deriva o índice AP. A tabela 1 apresenta a classificação da atividade geomagnética em função de valores de Kp e Ap.

Tabela 1 - Classificação da atividade geomagnética em função de Kp e Ap. Fonte: Batista (2003)

\begin{tabular}{c|c|c}
\hline Atividade Geomagnética & Índice Kp & Índice Ap \\
\hline Calma & $0-2$ & $<8$ \\
\hline Transição & 3 & $8-15$ \\
\hline Ativo & 4 & $16-29$ \\
\hline Tempestade Fraca & 5 & $30-49$ \\
\hline Tempestade Intensa & 6 & $50-99$ \\
\hline Tempestade muito intensa & $7-9$ & $100-400$ \\
\hline
\end{tabular}

Outro índice utilizado para a descrição de eventos solares é o índice Dst (Disturbance Storm Time). Esse índice é dado em nanotesla (nT) e representa o invólucro das curvas de medidas magnetométricas obtidas por uma cadeia de magnetrômetros localizados na região equatorial, ao longo do globo terrestre (YAMASHITA, 1999). É esse o parâmetro que melhor define a tempestade geomagnética, pois, antes e após a ocorrência de uma tempestade, o índice Dst tem sempre um valor padrão. Antes do início da tempestade, o índice Dst apresenta um "leve" pico de intensidade que é conhecido por "fase inicial" ou "início súbito". Após esse pico, desenvolve-se a "fase principal" da tempestade, que é caracterizada pela brusca queda do valor da intensidade do índice. Após alcançar o mínimo, o índice começa a subir de valor, essa fase é conhecida como "fase de recuperação", até atingir aproximadamente o valor calmo, que ocorre quando não há tempestade 
(YAMASHITA,1999).

A classificação da tempestade geomagnética em função desse índice é dada pela tabela a seguir (FEDRIZZI, 2003):

Tabela 2 - Classificação da atividade geomagnética em função de Dst. Fonte: Fedrizzi (2003)

\begin{tabular}{c|c}
\hline $\begin{array}{c}\text { Condição da Tempestade } \\
\text { Geomagnética }\end{array}$ & Valor do índice Dst (nT) \\
\hline Fraca & $-50<$ Dst $\leq-30$ \\
\hline Moderada & $-100<$ Dst $\leq-50$ \\
\hline Intensa & $-250<$ Dst $\leq-100$ \\
\hline Muito Intensa & $\leq-250$ \\
\hline
\end{tabular}

\section{EXPERIMENTOS, RESULTADOS E ANÁLISES}

No dia 20 de novembro de 2003 se iniciou uma intensa tempestade geomagnética às 08:03 UT (Hora Universal) como resultado de uma ejeção de massa coronal (CME) ocorrida na região ativa solar 501, em 18 de novembro de 2003. Em decorrência da CME, a velocidade do vento solar atingiu cerca de 700 $\mathrm{km} / \mathrm{s}$, e houve forte componente para o sul do campo magnético interplanetário, o que induziu uma das mais intensas tempestades geomagnéticas do ciclo solar 23 (GOPALASWAMY, et al., 2005; BECKER-GUEDES et al., 2007).

Para descrever a intensa tempestade geomagnética gerada em decorrência da ejeção de massa coronal foram gerados gráficos de valores dos índices geomagnéticos $\mathrm{Kp}$ e Dst. O gráfico de índice Dst foi elaborado para todo mês de novembro de 2003 com o objetivo de caracterizar o período de ocorrência da tempestade geomagnética em comparação com os dias sem a intensa tempestade. A figura 1 mostra os valores de índice Dst para o mês de novembro de 2003.

$\mathrm{Na}$ figura 1 é possível observar a ocorrência de uma intensa tempestade geomagnética no dia 20 de novembro, pois está caracterizada no gráfico a brusca queda do valor de DST, alcançando um mínimo, e então, aumentando até atingir valores calmos, quando não há mais tempestade. $\mathrm{O}$ valor de índice Dst atingido nessa tempestade geomagnética é $-472 \mathrm{nT}$ às 20:00 UT. Esse valor de índice Dst caracteriza, de acordo com a tabela 2, a ocorrência de uma tempestade geomagnética muito intensa.

A figura 2 mostra os gráficos de valores do índice Kp para cada dia do período de 18 até 23 de novembro de 2003 . Os intervalos horários para cada dia estão em Hora Universal (UT).

A partir da figura 2 se observa que o valor máximo do índice Kp ocorreu entre às 15:00 UT e 18:00 UT do dia 20 de novembro, atingindo o valor máximo da escala desse índice (9), o que caracteriza a ocorrência de uma tempestade muito intensa, de acordo com a tabela 1. 
Figura 1 - Índice geomagnético Dst para o mês de novembro de 2003.

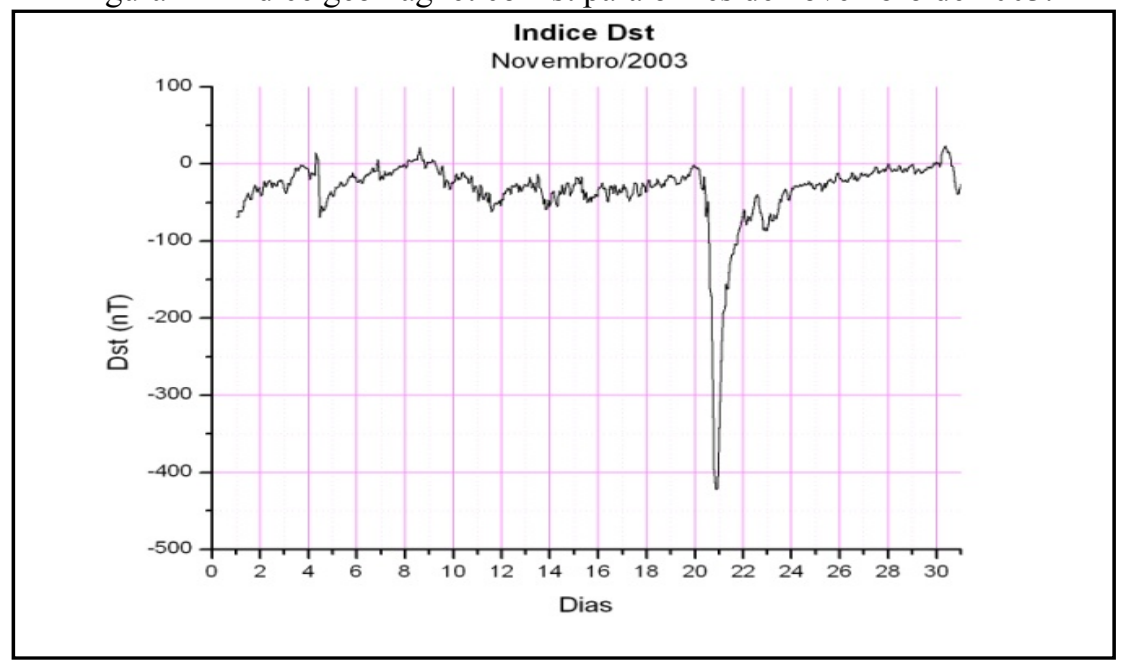

Figura 2 - Índice geomagnético Kp para o período de 18 até 23 de novembro de 2003.

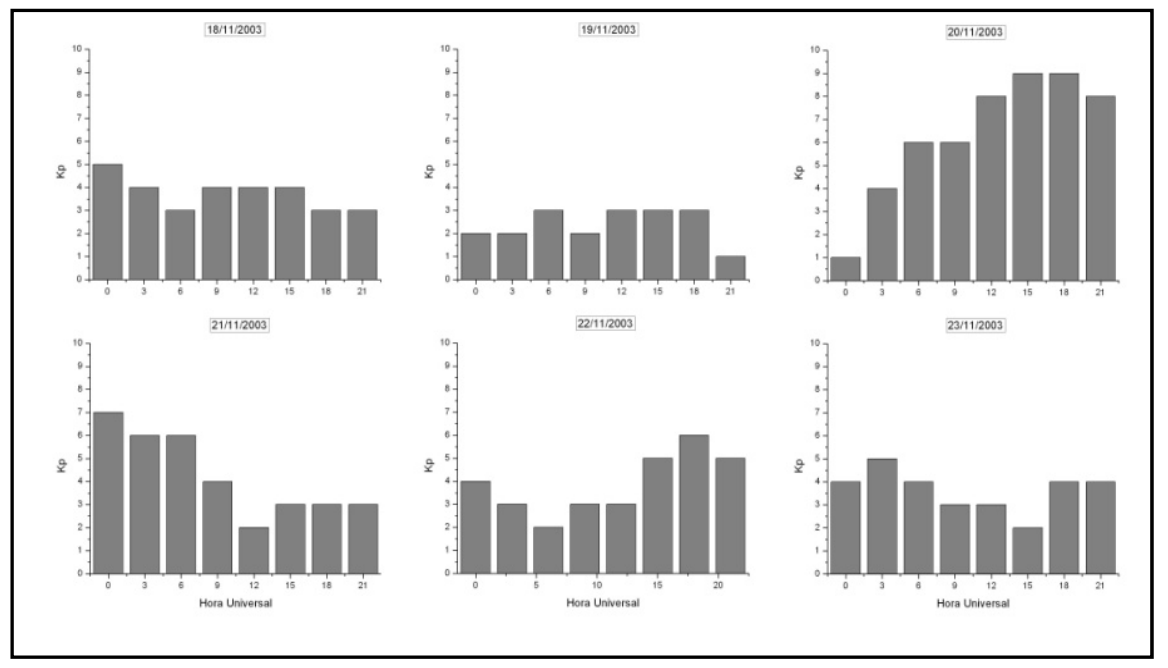

\subsection{Dados Utilizados}

Os experimentos que foram realizados utilizaram arquivos que contém grades de valores de VTEC (Vertical TEC - TEC na direção vertical) dos Mapas Globais da Ionosfera (arquivos no formato IONEX), disponibilizados pelo IGS (International GNSS Service).

Bol. Ciênc. Geod., sec. Artigos, Curitiba, v. 19, no 1, p.14-33, jan-mar, 2013. 
Para o estudo do posicionamento com GPS e para calcular o VTEC e os índices de irregularidades foram utilizados os dados das estações: BOMJ (Bom Jesus da Lapa/BA), BRAZ (Brasília/DF), CRAT (Crato/CE), CUIB (Cuiabá/MT), FORT (Fortaleza/CE), IMPZ (Imperatriz/MA), PARA (atualmente, UFPR) (Curitiba/PR), POAL (Porto Alegre/RS), RECF (Recife/PE), SALV (Salvador/BA), SMAR (Santa Maria/RS), UEPP (atualmente, PPTE) (Presidente Prudente/SP) e VICO (Viçosa/MG), pertencentes à RBMC (Rede Brasileira de Monitoramento Contínuo). Todos os dados foram processados e analisados e se referem ao período de 18 até 23 de novembro de 2003, que abrange o dia que ocorreu a tempestade geomagnética. Devido ao grande volume de resultados gerados, no artigo foram selecionados os resultados das estações IMPZ, UEPP e POAL dos dias 19, 20 e 21 de novembro de 2003 para serem apresentados, de tal forma que representam, sem nenhum prejuízo, as conclusões obtidas no estudo.

\subsection{Resultados e Análises}

Os arquivos IONEX do IGS fornecem valores de VTEC para todo o globo terrestre em uma grade diária com resolução espacial de $5^{\circ}$ em longitude por $2,5^{\circ}$ em latitude, e com resolução temporal de 2 horas (SCHAER, 1999). A partir desses arquivos foram gerados mapas globais da ionosfera para cada dia do período de 18 até 23 de novembro de 2003, para cada intervalo horário de 2 horas.

Neste artigo, selecionaram-se para apresentação nas figuras 3 e 4 os mapas globais da ionosfera das $08,12,16$ e 20 horas UT para os dias 19 e 20 de novembro de 2003, dia anterior e dia da tempestade, respectivamente.

Figura 3 - Mapas Globais da Ionosfera para o dia 19 de novembro de 2003.
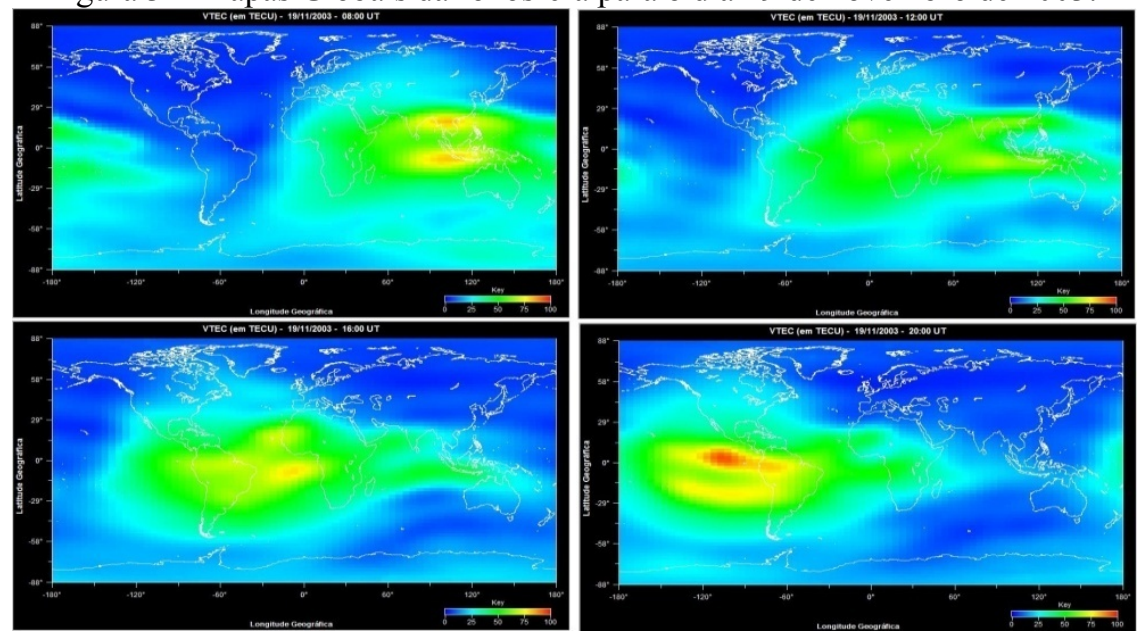
Figura 4 - Mapas Globais da Ionosfera para o dia 20 de novembro de 2003.
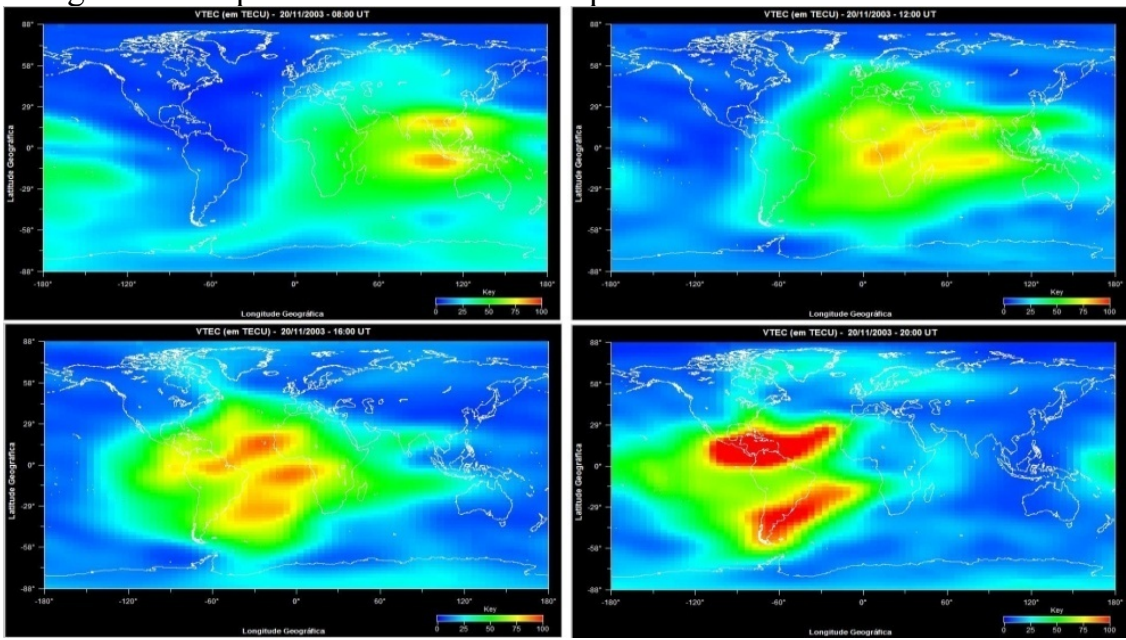

A partir das figuras 3 e 4 é possível realizar uma análise comparativa entre o dia da tempestade e o dia anterior a mesma. Desta forma, se constata o aumento nos valores de VTEC, consequentemente da densidade de elétrons livres, em regiões próximas ao equador geomagnético a partir das 16:00 UT, aproximadamente, do dia da tempestade, além da intensificação do efeito fonte que desloca elétrons para regiões ainda mais afastadas do equador geomagnético. As cristas norte e sul da anomalia equatorial, além de possuírem maior densidade de elétrons no dia da tempestade, também se deslocaram para regiões mais afastadas do equador geomagnético do que em dias geomagneticamente calmos.

Com os dados GPS foram obtidos os valores de VTEC de cada estação utilizando um programa computacional desenvolvido por Camargo (1999), que foi originalmente elaborado para modelagem e correção do erro ionosférico nas observáveis GPS, e adaptado por Matsuoka (2007) para finalidade de produzir como saída valores calculados de VTEC. O método empregado para o cálculo do VTEC a partir de dados GPS pode ser verificado em Matsuoka e Camargo (2004).

Foram elaborados gráficos de VTEC para cada uma das 13 estações, para cada dia do período de 18 até 23 de novembro de 2003. Devido à grande quantidade de gráficos gerados serão apresentados somente os resultados para algumas estações e para os dias 19, 20 e 21 de novembro, que sumarizam de forma adequada o que ocorreu. Neste sentido, a figura 5 mostra os gráficos obtidos para as estações IMPZ, UEPP e POAL para o dia anterior, o dia da tempestade e o dia posterior a mesma. As estações apresentadas foram escolhidas para mostrar o impacto da tempestade geomagnética, sendo que a localização das mesmas varia com a latitude: a estação IMPZ mais próxima ao equador geomagnético; a estação POAL mais ao sul e a 
UEPP localizada dentro da região que normalmente engloba parte da crista sul da anomalia equatorial.

Figura 5 - VTEC calculado para as estações IMPZ, UEPP e POAL para os dias 19, 20 e 21 de novembro de 2003.

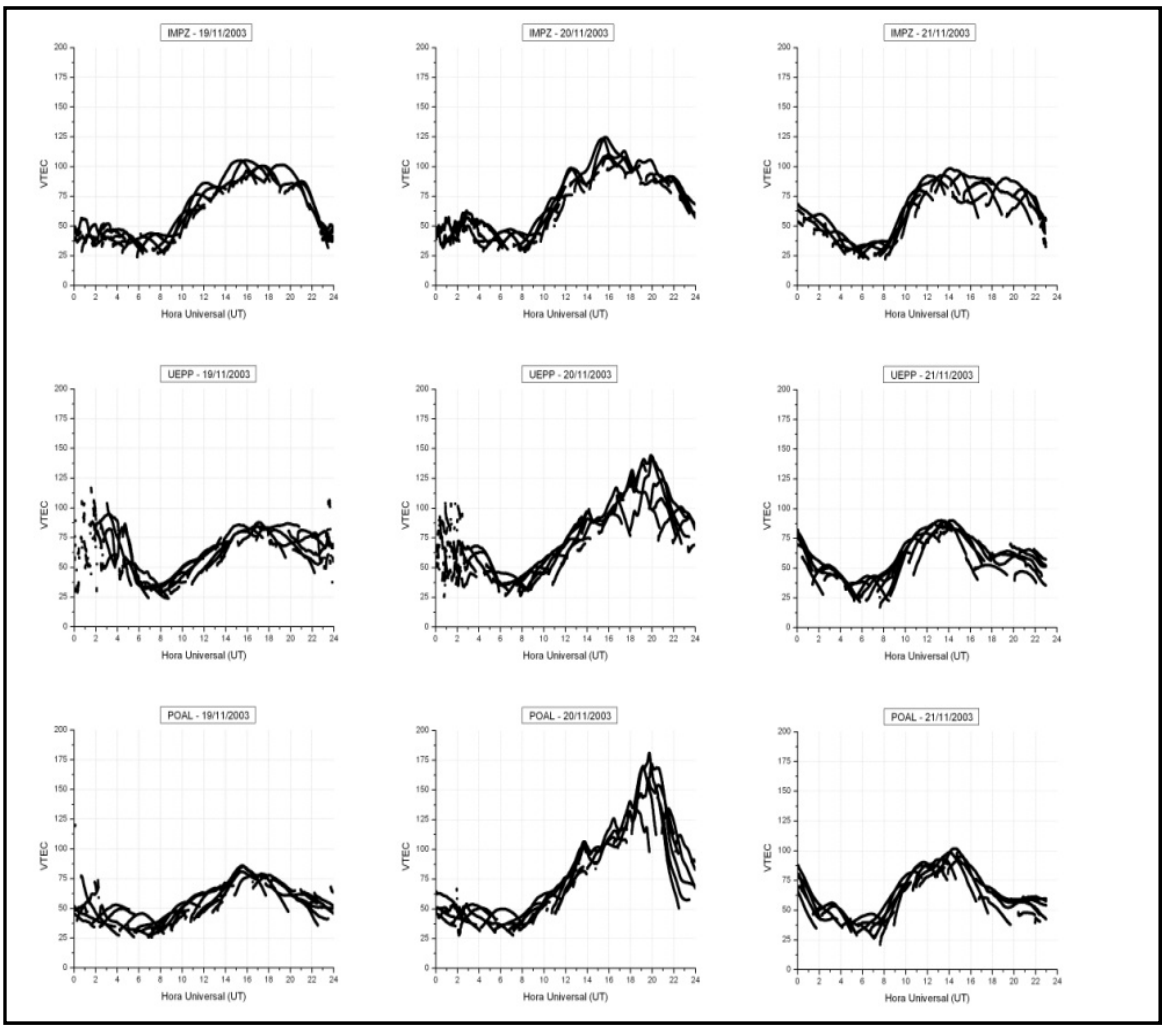

A partir dos gráficos de VTEC dos dias 19, 20 e 21 de novembro é possível fazer uma comparação do dia da tempestade (20/novembro) com o dia anterior e posterior a mesma. Analisando os gráficos para cada uma das estações, pode-se observar que houve um aumento na densidade de elétrons livres nas regiões mais próximas do equador geomagnético, no período da tarde no dia da tempestade (ver VTEC da estação IMPZ - aumento em torno de 20\% por volta das 15:00-16:00 UT quando comparado com o mesmo horário do dia anterior). Este aumento observado em IMPZ é relativamente menor do que os que foram observados para as demais estações mais afastadas do equador geomagnético. Neste contexto, para a estação UEPP, por volta das 20:00 UT, o aumento de VTEC no dia da tempestade quando comparada com o mesmo horário do dia anterior foi de aproximadamente $70 \%$. 
O aumento é ainda mais destacado analisando o VTEC para a estação POAL, pois, verificou-se para o dia da tempestade geomagnética, um deslocamento dos elétrons livres para regiões mais afastadas do equador geomagnético, após o pôr do Sol, em decorrência do efeito fonte intensificado, comum em períodos de tempestade geomagnética muito intensa.

Após as 20:00 UT do dia 20 de novembro, se observa que a crista sul da anomalia equatorial se deslocou para regiões de latitudes mais afastadas do equador geomagnético, atingindo valores máximos de VTEC nas estações GPS que se localizam mais ao sul, como a estação POAL. Por volta das 20:00 UT o aumento do VTEC nesta estação no dia da tempestade quando comparado com o dia anterior foi de aproximadamente $140 \%$.

Nos gráficos de VTEC para o dia posterior à tempestade (21/novembro) os aumentos relatados não são verificados, estando muito mais próximo ao comportamento do dia anterior da tempestade, evidenciando que os aumentos de VTEC do dia 20 estão relacionados à tempestade geomagnética ocorrida.

Os dados GPS diários das estações pertencentes à RBMC foram processados no programa GPSPACE 3.3 (GPS Positioning from ACS Clocks and Ephemerides) do NRCan (Natural Resource Canadá). No processamento foram utilizadas as pseudodistâncias advindas do código C/A em L1, coletadas com ângulo de elevação superior a 15 graus, arquivos de efemérides precisas e correções precisas dos relógios dos satélites e GDOP (Geometric Dilution of Precision - Diluição da precisão geométrica) menor que 7. Para correção do erro troposférico utilizou-se o modelo de Hopfield. Nenhum modelo para correção do erro devido à ionosfera foi utilizado. Com esses procedimentos procurou-se minimizar os diversos erros envolvidos no posicionamento, deixando predominante a influência da ionosfera sobre os resultados. As coordenadas foram estimadas a cada 15 segundos e então comparadas com as coordenadas conhecidas das estações, e desta forma, os valores de erro em planimetria e altitude geométrica foram obtidos.

As figuras a seguir mostram os gráficos de erro em planimetria e altimetria para as estações IMPZ, UEPP e POAL para os dias 19, 20 e 21 de novembro de 2003.

A significativa variação temporal da discrepância para a estação POAL demonstra o efeito causado pelo deslocamento da crista sul da anomalia equatorial para regiões mais afastadas do equador geomagnético, ocorrido no dia da tempestade. Conforme a análise dos gráficos apresentados, em altitude geométrica é verificada um comportamento temporal irregular em discrepância em todas as estações GPS no dia da tempestade, o que mostra a influência da intensa tempestade geomagnética do dia 20 de novembro de 2003 no posicionamento por ponto com GPS. 
Figura 6 - Erro em planimetria e altitude geométrica para estação IMPZ

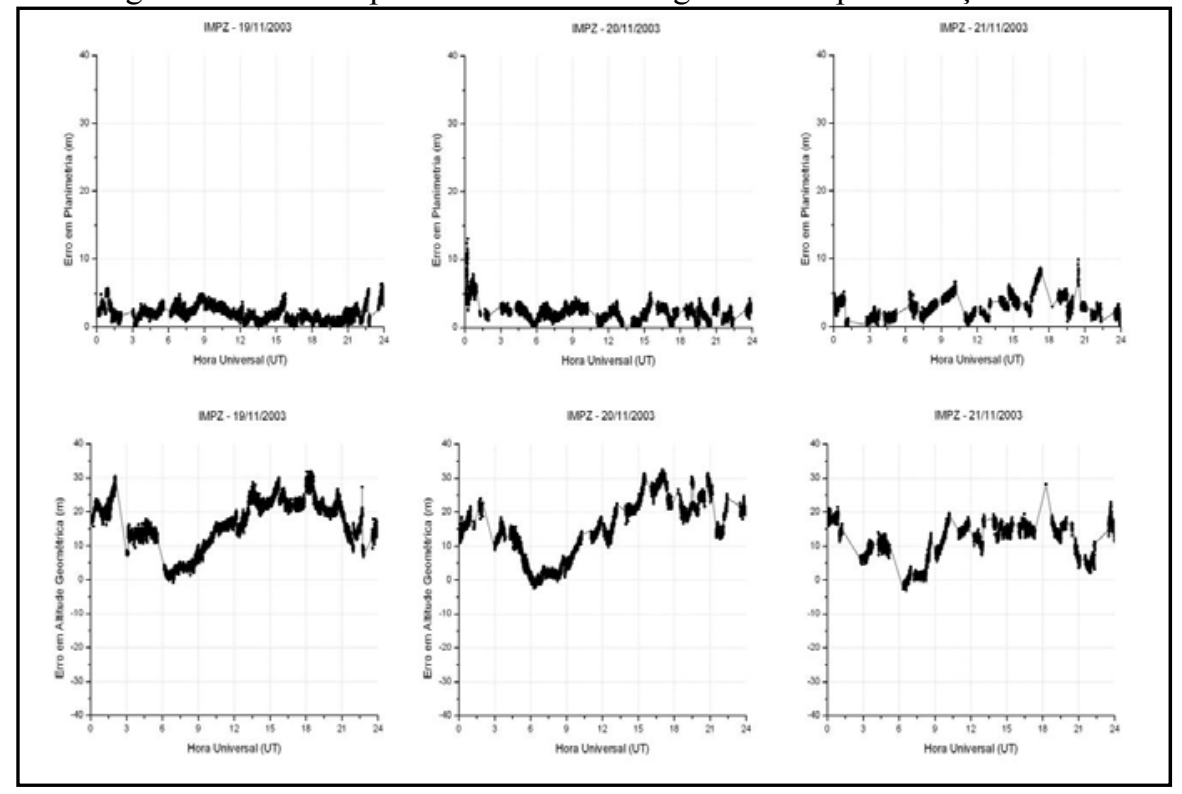

Figura 7. Erro em Planimetria e Altimetria para estação UEPP.

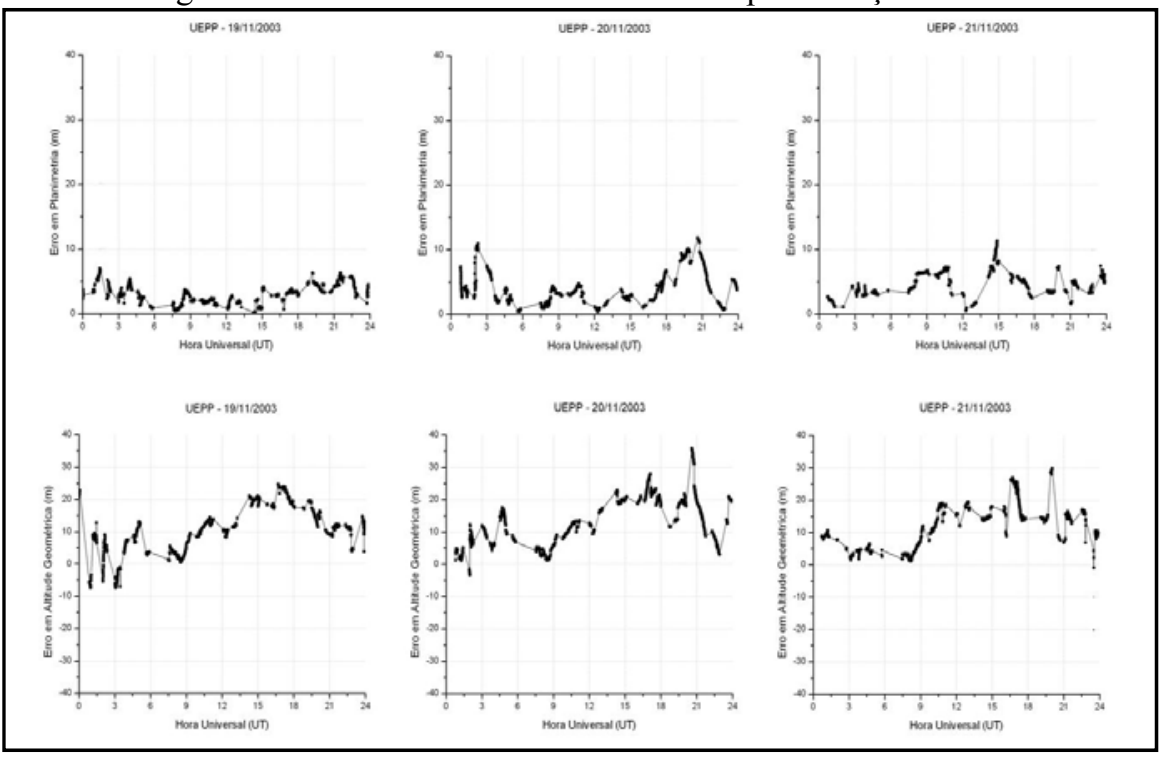

Bol. Ciênc. Geod., sec. Artigos, Curitiba, v. 19, nº 1, p.14-33, jan-mar, 2013. 
Figura 8. Erro em Planimetria e Altimetria para estação POAL.

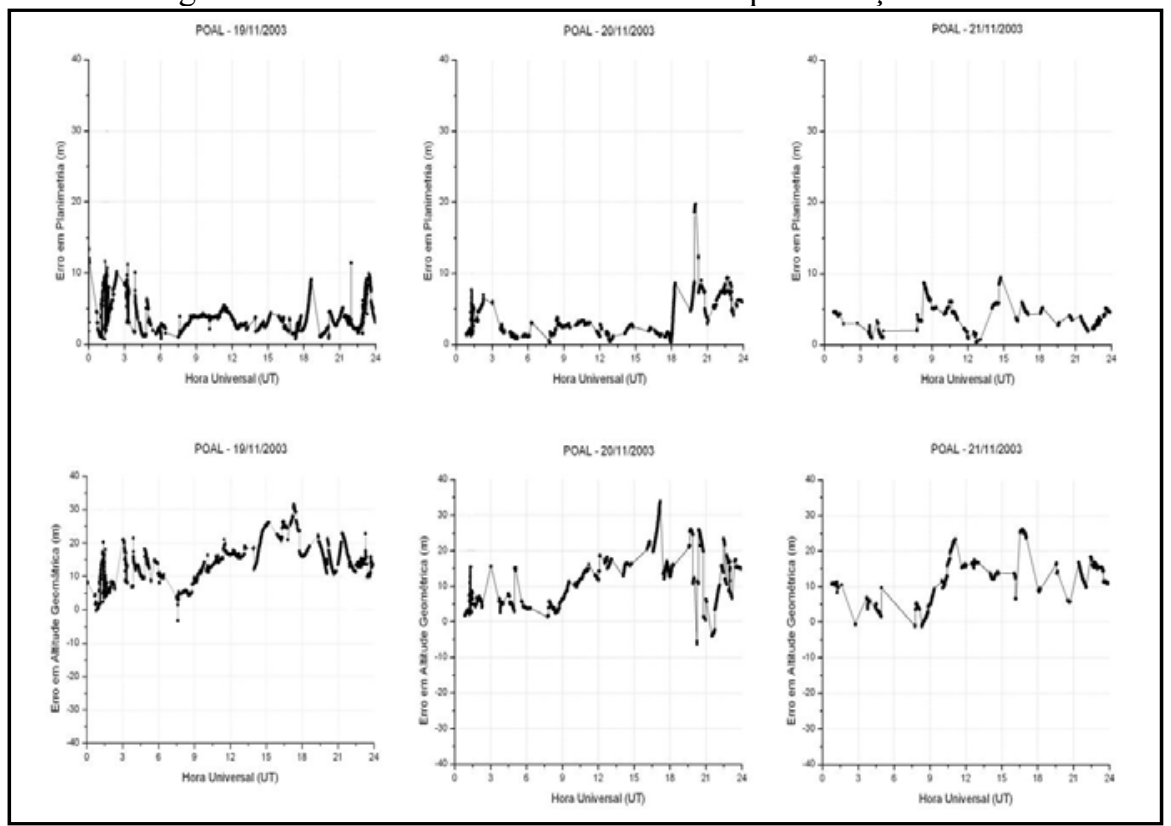

Analisando os gráficos de erro no posicionamento, bem como, confrontando-os com os do VTEC, pode-se verificar os impactos da intensa tempestade geomagnética no posicionamento com GPS na região brasileira.

Nos gráficos de erro em planimetria para a estação IMPZ não se verifica um impacto nos valores para o dia da tempestade com relação aos dias anterior e posterior, considerados calmos. Mas com relação às discrepâncias na componente altimétrica, se verifica um aumento nos valores a partir das 18:00 UT do dia 20 de novembro. Com relação à estação UEPP, verifica-se maiores discrepâncias em planimetria e altimetria a partir das 18:00 UT do dia da tempestade, em comparação com os demais dias.

No gráfico da estação POAL referente ao erro planimétrico se verifica aumento nos valores a partir das 18:00 UT do dia 20 de novembro com relação aos dias anterior e posterior a tempestade geomagnética. E com relação à altimetria, verificase aumento nas discrepâncias também a partir do mesmo horário. Os valores de discrepâncias verificados para essa estação são maiores que os observados para as outras estações, o que evidencia a influência do aumento do VTEC e o maior deslocamento da crista sul da anomalia equatorial que ocorreu no dia da tempestade e pode ser verificado nos gráficos de VTEC já apresentados.

Para finalizar, um estudo sobre irregularidades ionosféricas também foi realizado para verificar se houve mudanças devido à tempestade geomagnética. 
Com os dados de TEC das estações GPS foram calculadas a taxa de variação do TEC, e a partir deles foi obtido um índice de irregularidades denominado de $F_{p}$. Esse índice foi proposto por Mendillo et al. (2000), possui resolução horária e é calculado para cada estação GPS. É um indicador do nível geral de irregularidades ionosféricas na região da estação. O processamento dos dados para a obtenção do índice $\mathrm{F}_{\mathrm{p}}$ foi realizado utilizando o software desenvolvido pelo Grupo de Estudos de Geodésia Espacial da Unesp, denominado, ION_INDEX (PEREIRA e CAMARGO, 2012).

A figura 9 apresenta os valores do índice $F_{p}$ para as estações IMPZ, UEPP e POAL para os dias 19, 20 e 21 de novembro de 2003.

Figura 9 - Índice de Irregularidades ionosféricas $\mathrm{F}_{\mathrm{p}}$ para IMPZ, UEPP e POAL.

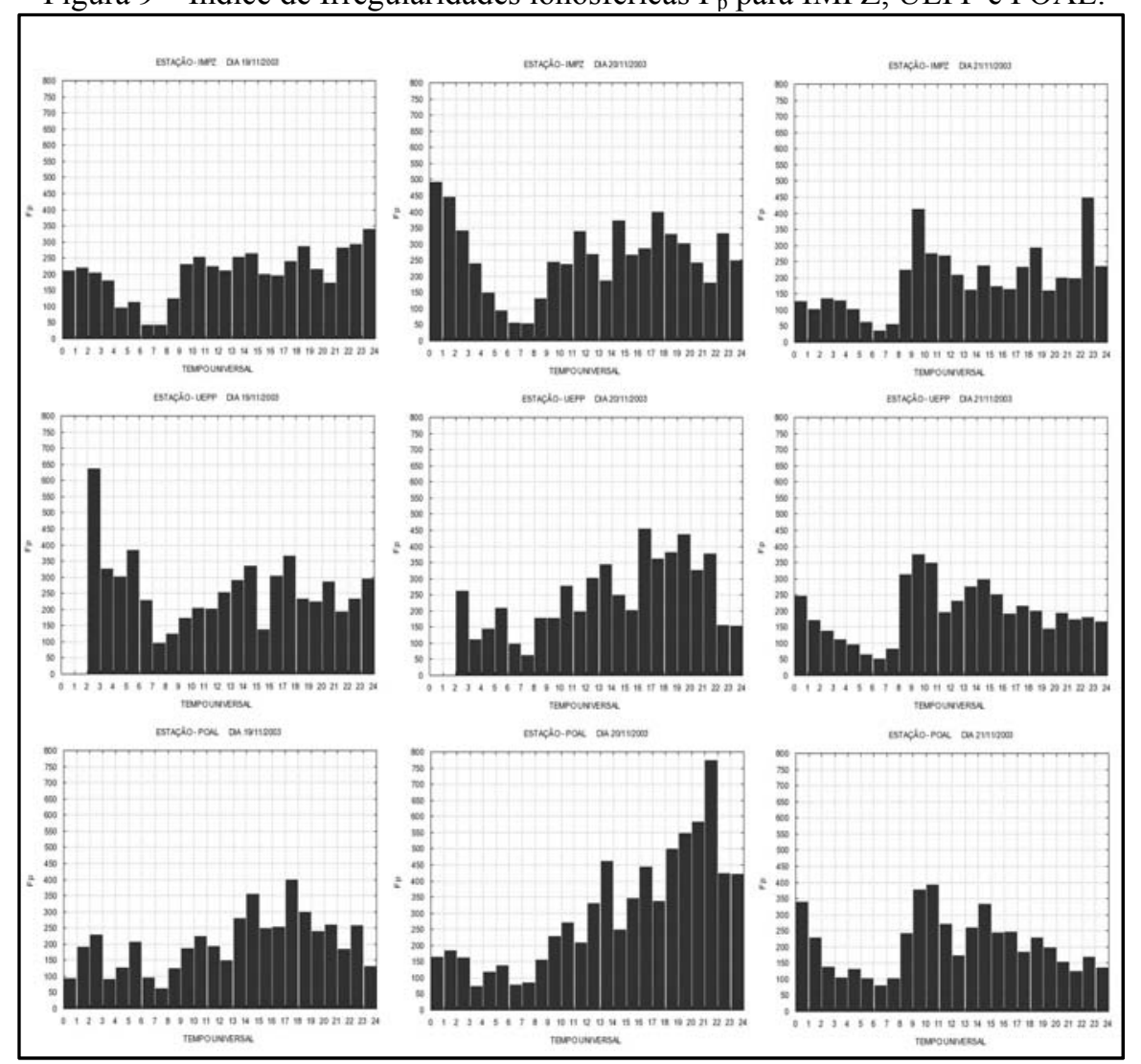

Segundo Mendillo et al. (2000), valores de $\mathrm{F}_{\mathrm{p}} \leq 50$ representam baixos níveis de irregularidades; $50<\mathrm{F}_{\mathrm{p}} \leq 200$ significam a presença de moderadas irregularidades e 
$\mathrm{F}_{\mathrm{p}}>200$ representam a ocorrência de níveis de irregularidades muito intenso. Analisando a figura 9, pode-se verificar de forma geral valores altos de $F_{p}$ o que está de acordo com o se espera para o mês de novembro em período de alta atividade solar. Focando no objetivo do artigo, a principal conclusão que se obtém ao analisar os valores de índices $F_{p}$ é o aumento observado para a estação POAL no dia da tempestade geomagnética em comparação aos outros dias. Isto está em concordância com o deslocamento da crista sul da anomalia equatorial observado nos valores de VTEC para a estação POAL (figura 5), e que, com base então nos valores de $F_{p}$ indicaram também que pode ter ocorrido um aumento do nível de irregularidades ionosféricas na região Sul do Brasil, que possivelmente teve influência nas mudanças observadas e já analisadas para dia da tempestade nos resultados do posicionamento da estação POAL (figura 8).

\section{CONCLUSÕES}

Nesse artigo foi realizado um estudo do impacto da intensa tempestade geomagnética de 20 de novembro de 2003 no comportamento do VTEC, do erro no posicionamento por ponto com GPS e de irregularidades ionosféricas na região brasileira utilizando dados GPS da RBMC.

Os resultados mostraram um aumento do VTEC na região brasileira no período da tarde do dia da tempestade, principalmente em regiões próximas ao equador geomagnético. Mas o que mais se destaca, ao se comparar o dia da tempestade com os demais dias analisados, é o aumento observado no VTEC após o pôr do Sol no dia da tempestade em regiões mais ao Sul do Brasil, sugerindo um deslocamento da crista da anomalia equatorial para latitudes mais afastadas do equador, indicando um possível aumento do efeito fonte na região equatorial. Os resultados também indicaram um aumento do nível de irregularidades ionosféricas no dia da tempestade, principalmente para a região Sul, concordando com o aumento do TEC ocorrido na região.

Diante deste cenário de aumento do TEC e das irregularidades no dia da tempestade, verificou-se nos resultados do posicionamento por ponto que as maiores variações e magnitudes, decorrentes da tempestade, ocorreram na região mais ao sul do Brasil, sendo mais detectados na estação POAL, principalmente para o período após as 18:00 UT. Nas outras estações utilizadas no experimento, também se verificou uma variação temporal irregular do erro no posicionamento quando comparado o dia da tempestade com os demais dias, porém com menor impacto.

As mudanças ocorridas no comportamento do TEC, nas irregularidades ionosféricas e no posicionamento por GPS no dia da tempestade geomagnética caracterizam a influência que uma intensa tempestade geomagnética pode provocar na região brasileira. Com a chegada do período de alta atividade do ciclo solar 24, espera-se o aumento dos fenômenos solares, e consequentemente um aumento das ocorrências de tempestades geomagnéticas intensas, e estudos como relatados neste artigo ajudam a entender o que se pode esperar, embora cada caso possa ter suas 
peculiaridades, sugerindo, portanto, a importância da continuação dos estudos dos efeitos das tempestades geomagnéticas no GNSS no Brasil.

\section{AGRADECIMENTOS}

Os autores agradecem aos apoios financeiros: Projeto Universal CNPq (proc. n. 481339/2010-8), Projeto Temático Fapesp (proc. n. 2006/04008-2), Bolsa de IC PROBIC/FAPERGS (2011/2012), Bolsa de Doutorado CAPES/FAPERGS (2012), Bolsas de Produtividade em Pesquisa CNPq (Proc.n. 307472/2009-4 e Proc.n. 306719/2010-0) e Bolsa IC Fapesp (2011/00986-8). Agradecimentos ao IBGE pelos dados GNSS da RBMC e ao GSD/NRCan pelo software GPSPACE.

\section{REFERÊNCIAS BIBLIOGRÁFICAS}

AFRAIMOVICH, E.L.; ALTYNSEV, A.T.; GRECHNEV, V.V.; LEONOVICH, L.A.. Ionospheric Effects of the solar flares as deduced from global GPS network data. Advances in Space Research, v.27, n.6-7, p.1333-1338, 2001.

APPLETON, E.V. Two anomalies in the ionosphere. Nature, v.157, 691, 1946.

BATISTA, I.S.. Introdução à ionosfera e a propagação de ondas (Notas de Curso Ministrado). 2003. Programa de Pós-Graduação em Ciências Cartográficas, Faculdade de Ciências e Tecnologia, Universidade Estadual Paulista.

BECKER-GUEDES, F., SAHAI Y., FAGUNDES, P. R., ESPINOZA E. S., PILLAT V. G., LIMA W. L. C., BASU SU., OTSUKA Y., SHIOKAWA K., MACKENZIE E. M., PI W., BITTENCOURT J. A. The ionospheric response in the Brazilian sector during the super geomagnetic storm on 20 November 2003. Annales Geophysicae, v 25. p 863-873, 2007.

CAMARGO, P.O.. Modelo regional da ionosfera para uso em posicionamento com receptores de uma freqüência. 1999. 191p. Tese (Doutorado em Ciências Geodésicas) - Setor de Ciências da Terra, Universidade Federal do Paraná, Curitiba.

CAMPOS, M.A.; WANNINGER, L.; SEEBER, G.. Condições ionosféricas perturbadas $e$ os sinais GPS. In: III Congresso Internacional da Sociedade Brasileira de Geofísica - Resumos Expandidos, Rio de Janeiro/RJ, p.601-604, 7 a 11 de novembro, 1993.

DAVIES, K.. Ionospheric Radio. London: Peter Peregrinus Ltd., 1990. 580p.

FEDRIZZI, M.. Estudo do efeito das tempestades magnéticas sobre a ionosfera utilizando dados do GPS. 2003. 223p. Tese (Doutorado em Geofísica Espacial) - Instituto Nacional de Pesquisas Espaciais, São José dos Campos.

GONZALEZ, W.D.; TSURUTANI, B.T.; GONZALEZ, A.L.C.. Interplanetary origin of geomagnetic storms. Space Science Reviews, v.88, p.529-562, 1999.

GOPALASWAMY, N., YASHIRO, S., MICHALEK, G., XIE, H., LEPPING, R. P., AND HOWARD, R. A.: Solar source of the largest geomagnetic storm of cycle 23, Geophysical Research Letter, v.32, L12S09, doi:10.1029/2004GL021639, 2005. 
HATHAWAY, D. H. Coronal Mass Ejections. 2007. Dísponível em: http://solarscience.msfc.nasa.gov/SunspotCycle.shtml.

ISGI (International Service of Geomagnetic Indices) - online - endereço eletrônico: http://www.cetp.ipsl.fr/ isgi/indicgeo.htm, acesso em março de 2011.

KIRCHHOFF, V.W.J.H.. Introdução à geofísica espacial. São Paulo: Nova Stella, Ed. USP/FAPESP, 1991. 149p.

KOMJATHY, A.; SPARKS, L.; MANNUCCI, A.J.; XIAOQING, P.. An assessment of the current WAAS ionospheric correction algorithm in the south American region. Journal of the Institute of Navigation, v.50, n.3, p.193-218, Fall/2003.

LIU, J. Y., LIN, C.H.; CHEN, Y.I.; LIN, Y.C.; FANG, T.W.; CHEN, C.H.; CHEN, Y. C.; HWANG, J.J.. Solar flare signatures of the ionospheric GPS total electron content. Journal of Geophysical Research, v.111, A05308, doi:10.1029/2005JA011306, 2006.

MATSUOKA, M.T.; CAMARGO, P.O.. Cálculo do TEC usando dados de receptores GPS de dupla freqüência para a produção de mapas da ionosfera para a região brasileira. Revista Brasileira de Cartografia, n.56/01, p.14-27, julho/2004.

MATSUOKA, M.T.; CAMARGO, P.O.; BATISTA, I.S.. Impacto de explosões solares no comportamento da ionosfera e no posicionamento com GPS na região brasileira: Estudo de caso para o dia 28 de outubro de 2003. Boletim de Ciências Geodésicas, v.12, n.2, p.315-334, 2006.

MATSUOKA, M.T.. Influência de diferentes condições da ionosfera no posicionamento por ponto com GPS: Avaliação na região brasileira. 2007. 263p. Tese (Doutorado em Ciências Cartográficas) - Programa de PósGraduação em Ciências Cartográficas, Faculdade de Ciências e Tecnologia, Universidade Estadual Paulista, Presidente Prudente.

MATSUOKA, M.T.; CAMARGO, P.O.; BATISTA, I.S.. Posicionamento por GPS na região Brasileira durante a intensa tempestade geomagnética de 29 de outubro de 2003. Pesquisas em Geociências, v.35, n.1, p.3-19, 2008.

McNAMARA, L.F.. The ionosphere: communications, surveillance, and direction finding. Florida: Krieger Publishing Company, 1991. 237p.

MENDILLO, M.; LIN, B.; AARONS, J. The application of GPS observations to equatorial aeronomy. Radio Science, v. 35, n.3, p.885-904, 2000.

PEREIRA, V.A.S.; CAMARGO, P.O.. Índices de irregularidades da ionosfera obtidos a partir das observáveis GNSS. In: Anais do III Simpósio Brasileiro de Geomática, FCT/Unesp, Presidente Prudente-SP, p.286-291, 2012.

PI, X.; MANNUCCI, A. J.; LINDQWISTER, U. J.; HO, C. M. Monitoring of Global Ionospheric Irregularities Using the Worldwide GPS Network, Geophysical Research. Letters, v.24, n.18, 2283-2286, 1997.

RODRIGUES, F.S.R.. Estudo das irregularidades ionosféricas equatoriais utilizando sinais GPS. 2003. 151p. Dissertação (Mestrado em Geofísica Espacial) - Instituto Nacional de Pesquisas Espaciais, São José dos Campos. 
SALOMONI, C.S.; MATSUOKA, M.T.; SOUZA, S.F.. Efeitos de tempestades geomagnéticas no posicionamento relativo com GPS na região brasileira: estudo de caso para 29 de outubro de 2003. Revista Brasileira de Cartografia, v.63, p.449-459, 2011.

SANDFORD, W.H.. The impact of solar winds on navigation aids. Journal of Navigation, v.52, issue 1, 42-46, jan., 1999.

SCHAER, S. Mapping and predicting the earth's ionosphere using the Global Positioning System. 1999. 205p. Ph.D. dissertation. University of Bern, Bern, Switzerland.

SKONE, S., K. KNUDSEN, M. DE JONG, Limitations in GPS receiver tracking performance under ionospheric scintillation conditions, Phys. Chem. Earth (A), 26, 613-621, 2001.

WANNINGER, L. Ionospheric Monitoring Using IGS Data, paper presented at the IGS Workshop, Inst. of Geol. Sci., Bern, Switzerland, March 1993.

WANNINGER, L.; SEEBER, G.; CAMPOS, M.A.. Use of GPS in the south of Brazil under severe conditions. In: IAG Symposium III, Heidelberg, 1991.

WEBSTER, I.. A Regional Model for Prediction of Ionospheric Delay for Single Frequency Users of the Global Positioning System. 1993. M.Sc.E Thesis Department of Surveying Engineering, University of New Brunswick, New Brunswick, Canada.

YAMASHITA, C.S.. Efeito das tempestades magnéticas intensas na ionosfera de baixa latitude. 1999. 75p. Dissertação (Mestrado em Geofísica Espacial) Instituto Nacional de Pesquisas Espaciais, São José dos Campos.

(Recebido em outubro de 2012. Aceito em dezembro de 2012.) 